\title{
Factores asociados a inadecuadas prácticas de distanciamiento social durante la pandemia por COVID-19 en Perú
}

\section{Factors associated to inadequate practices of social distancing during the COVID-19 pandemic in Peru}

$\mathrm{DOI}$

https://doi.org/10.35434/rcmhnaaa.2021.14Sup1.1154

\section{RESUMEN}

Objetivo: Determinar los factores asociados a inadecuadas prácticas de distanciamiento social durante la pandemia por COVID-19 en Perú. Material y métodos. Se administró una encuesta en línea dirigida para adultos de diversas regiones del Perú seleccionados mediante un muestreo por bola de nieve, la encuesta incluía una escala para la medición de prácticas de distanciamiento social, el uso de la información, el afrontamiento emocional frente al coronavirus y características sociodemográficas. Se realizó un análisis bivariado y multivariado utilizando razones de prevalencia cruda (RP) y ajustadas (RPa) con intervalos de confianza al $95 \%$ por medio de modelos lineales generalizados. Resultados. Se incluyeron 377 personas de los cuales el 55,4\% fueron hombres y $77,5 \%$ residían fuera de Lima, el $35 \%$ de los encuestados tuvo inadecuadas prácticas de distanciamiento social lo que estuvo asociado con tener primaria o secundaria incompleta (RPa 1,41, IC $95 \%: 1,11$ a 1,81), inadecuado uso de la información ( $\mathrm{RPa} 2,82$, IC 95 \%: 1,98 a 4,05) e inadecuado afrontamiento emocional frente al coronavirus (RPa 1,76, IC 95 \%: 1,27 a 2,42). Conclusión. Personas con menor grado de instrucción, inadecuado uso de la información y con un negativo afrontamiento emocional frente al coronavirus tienen mayor probabilidad de inadecuadas prácticas de distanciamiento social.

Palabras Clave: : Prevención y control; COVID-19; Adaptación Psicológica; Servicios de Información; Encuestas y Cuestionarios; Perú (Fuente: DeCSBIREME).

\section{ABSTRACT}

Objetive: To determine the factors associated with inappropriate practices of social distancing during the COVID-19 pandemic in Peru. Material and methods. It was conducted an online survey that included an scale for measuring practices of social distancing, the use of information and emotional coping with coronavirus and socio-demographic characteristics. A bivariate and multivariate analysis was performed using crude (RP) and adjusted (RPs) prevalence ratios with $95 \%$ confidence intervals using generalized linear models. Results. There were included 377 people, $35 \%$ of whom had inadequate practices of social distancing, which was associated with having incomplete primary or secondary education ( $\mathrm{RPa} 1.4195 \% \mathrm{Cl}: 1.11$ to 1.81 ) inadequate use of information ( $\mathrm{RPa} 2.8295 \% \mathrm{Cl}$ : 1.98 to 4.05 ) and inadequate emotional coping ( $\mathrm{RPa} 1.7695 \% \mathrm{Cl} 1.27$ to 2.42 ). Conclusion. Inappropriate practices of social distancing are more likely in people with less education, inadequate use of information and for emotional coping with the pandemic

Keywords: Prevention \& Control; COVID-19; Adaptation, Psychological; Information Services; Surveys and Questionnaires; Peru. (Source: DeCSBIREME).
Aníbal V. Díaz-Lazo, ${ }^{1, a}$, Mely Ruiz Aquino ${ }^{1, b}$, José Antonio Beraún-Barrantes ${ }^{1, c}$, Hugo Arroyo-Hernández ${ }^{2, c}$

FILIACIÓN

1. Universidad de Huánuco, Huánuco, Perú.

2. Oficina General de Información y Sistemas, Instituto Nacional de Salud, Lima, Perú.

Médico internista, Doctor en Medicina.

b. Licenciada en enfermería, magister en salud pública y gestión sanitaria.

c. Médico cirujano

ORCID

1. Aníbal V. Díaz-Lazo / 0000-0002-9282-9435

2. Mely Ruiz Aquino / 0000-0002-8340-7898

3. José Beraún-Barrantes / 0000-0001-8979-2734

4. Hugo Arroyo-Hernández / 0000-0001-5128-7820

CORRESPONDENCIA

Mely RuizAquino

EMAIL

melyruizaquino@udh.edu.pe

CONFLICTOS DE INTERÉS

Los autores declaran no tener conflictos de interés.

\section{FINANCIAMIENTO}

Este proyecto fue financiado por la Universidad de Huánuco, Huánuco, Perú.

\section{CONTRIBUCIÓN DE LOSAUTORES}

Todos los autores participaron en la concepción, diseño, interpretación de los datos, redacción del manuscrito, revisión crítica del manuscrito y aprobaron la version final.

\section{REVISIÓN DE PARES}

Recibido: $10 / 06 / 2021$

Aceptado: 22/08/2021

\section{COMO CITAR}

Diaz-Lazo, A., Ruiz-Aquino, M., Beraún-Barrantes, J., \& ArroyoHernández, H. Factores asociados a inadecuadas prácticas de distanciamiento social durante la pandemia por COVID-19 en Perú. Revista Del Cuerpo Médico Hospital Nacional Almanzor Aguinaga A s e n jo, 2021,14 ( S u p 1 ), 28 - 32 . https://doi.org/10.35434/rcmhnaaa.2021.14Sup1.1154
Esta obra está bajo una Licencia Creative

Commons Atribución 4.0 Internacional.

Versión Impresa: ISSN: 2225-5109

Versión Electrónica: ISSN: 2227-473

Cross Ref. DOI: 10.35434/rcmhnaaa

OJS: https://cmhnaaa.org.pe/ojs 


\section{INTRODUCCIÓN}

La pandemia de la enfermedad por coronavirus 2019 (COVID19) ha llevado a que los sistemas de salud en todos los países implementen acciones para mitigar la propagación del virus $^{(1,2)}$, siendo el confinamiento comunitario una de las acciones más drásticas que busca reducir las interacciones sociales entre personas ${ }^{(3)}$. Sumado a esto, se han propuesto nuevos comportamientos para el autocuidado por parte de los ciudadanos como son el uso de mascarillas, protector facial y el distanciamiento social. Esta última es una forma de prevención que consiste en alejarse de lugares concurridos y restringir la interacción entre las personas, tomando cierta distancia física o evitando el contacto directo entre ellas ${ }^{(4)}$.

Una revisión sistemática y metaanálisis para evaluar la protección personal frente a la COVID-19, el síndrome respiratorio agudo grave (SARS) o el síndrome respiratorio de oriente medio (MERS), muestra que una distancia física de al menos un metro está fuertemente asociada con la protección, pero distancias de hasta dos metros podrían ser más efectivas ${ }^{(5)}$. Las recomendaciones de distanciamiento físico no tienen mayores antecedentes en su aplicación a nivel latinoamericano y se desconoce si algunas características sociodemográficas, sociales o personales pudieran tener efecto en su práctica.

Por lo tanto, el presente estudio tuvo como objetivo determinar los factores asociados a las prácticas de distanciamiento social durante la pandemia por COVID-19 en Perú.

\section{MATERIALY MÉTODOS}

\section{Diseño y población de estudio}

Se realizó un estudio transversal y analítico mediante la aplicación de una encuesta en línea autoadministrada para ciudadanos procedentes de diversas regiones del Perú y que fueron seleccionados por medio de un muestreo no probabilístico. Mediante el método de bola de nieve se solicitó a cada participante replicar la invitación para realizar la encuesta entre sus contactos. Los criterios de inclusión fueron ser adultos de 18 a 60 años y el autorreporte de no haber sido diagnosticado con la COVID-19, mientras que debido a su trabajo cercano con personas sospechosas o enfermas de COVID-19 se excluyó a quienes manifestaron ser personal de salud.

\section{Instrumento de recolección de datos}

Para la elaboración de la encuesta se revisó la literatura existente e información publicada por la $\mathrm{OMS}^{(6)}$. La versión preliminar del contenido de la encuesta se sometió a una evaluación por dos jueces y cinco expertos en la temática del estudio. La encuesta fue administrada por Internet a través de las redes sociales y con acceso mediante la plataforma de Google Docs. El periodo de ejecución de la encuesta fue entre los meses de marzo y mayo del 2020.

\section{Variables}

La variable principal fue "la práctica de distanciamiento social”, la cual estuvo conformada por seis preguntas con respuestas en escala Likert que fueron: 1) Evita el contacto con sus vecinos, amigos, gente cercana que le rodea. 2 ) Mantiene al menos un metro y medio de distancia de cualquier persona. 3) Evita salir a la calle innecesariamente o lo hace solo en casos urgentes. 4) Se mantiene alejado, extremando medidas frente a personas sospechosas o con signos evidentes de coronavirus. 5) Evita las visitas a su casa. 6) Evita saludar convencionalmente (abrazo, beso, dar la mano).

Los factores asociados estuvieron conformados por variables independientes relacionadas con las características sociodemográficas de la población como el sexo (hombre o mujer), la edad (dicotomizada según la mediana), la región de residencia categorizada como en Lima o el Callao o fuera de Lima y el Callao, el nivel de instrucción alcanzada categorizada como completa para quienes terminaron la secundaria o superior o incompleta para quienes no terminaron la primaria o secundaria, así como el estado civil agrupado como con pareja si era casado o conviviente y sin pareja si era soltero o divorciado.

Se incluyeron además dos factores relacionados con el comportamiento durante la pandemia y que fueron el uso de la información sobre el coronavirus, considerado como adecuado e inadecuado, evaluadas mediante cuatro preguntas con respuestas en escala Likert, que fueron: 1) Se informa sobre las normas sanitarias emitidas por las autoridades de salud. 2) Evita informaciones falsas o alarmantes frente a la pandemia por el COVID-19. 3) Se informa de fuentes confiables sobre la situación de la enfermedad del coronavirus. 4) Evita la sobreinformación a propósito de la enfermedad del coronavirus. El siguiente factor fue el afrontamiento emocional frente al coronavirus, categorizado como adecuada o inadecuada, lo que generó tres preguntas con respuestas en escala Likert. 1) Aplica estrategias de afrontamiento al estrés o tensión en mi vida cotidiana. 2) Evita generar miedo respecto a la enfermedad del coronavirus a personas de mi entorno. 3) Piensa en positivo y me muestro optimista a pesar de las circunstancias. Según los puntajes para las variables que fueron medidas con una escala Likert (distanciamiento social, uso de la información y afrontamiento emocional), se generaron tertiles y estos fueron dicotomizados como adecuados para los puntajes dentro del tertil medio o superior y como inadecuados para el tertil inferior.

\section{Análisis estadístico}

En un primer momento, se realizó un análisis univariado mediante frecuencias y porcentajes para variables cualitativas y medidas de dispersión o tendencia central para variables cuantitativas. Seguido, se realizó un análisis bivariado y multivariado utilizando razones de prevalencia cruda (RP) y ajustada (RPa) con intervalos de confianza al 95 $\%$ por medio de modelos lineales generalizados con la familia Poisson y función de enlace log. Las variables con valores $\mathrm{p}<$ 0,05 se consideraron como estadísticamente significativos y fueron incluidas en los análisis ajustados. Se utilizó el paquete estadístico STATA 14.0.

\section{Aspectos éticos}

La finalidad y objetivos de la investigación fue informada a todos los participantes, siendo esta voluntaria y con 
posibilidad de abandonarlo en cualquier momento. El protocolo del estudio fue aprobado por el Comité de Ética en Investigación del Hospital Regional Docente Clínico Quirúrgico Daniel Alcides Carrión de Huancayo. Asimismo, el protocolo del estudio primario se registró en el repositorio PRISA del Instituto Nacional de Salud de Perú (código: El0000000875).

\section{RESULTADOS}

Se encuestaron un total de 377 personas; según sus características sociodemográficas el 53,3 \% tenían 30 años o más, el 55,4\% fueron hombres, el 77,5\% residían fuera de Lima, el 54,4\% tenían una pareja y el 85,2 \% reportaron tener secundaria o estudios superiores. Con respecto a las variables de comportamiento, el $29,4 \%$ tuvo un inadecuado uso de la información y el 28,4\% un afrontamiento emocional negativo frente a la pandemia (ver Tabla 1 ). El 35,0\% reportó tener practicas inadecuadas de distanciamiento social, las características de las preguntas que conformaron esta variable se presentan en la tabla 2.
Tabla 1. Características sociodemográficas y de comportamiento frente al coronavirus en adultos que participaron de una encuesta en línea durante marzo y mayo de 2020 en Perú.

\begin{tabular}{lcc} 
& $\mathrm{n}=377$ & $(\%)$ \\
$\begin{array}{l}\text { Edad } \\
\quad 30 \text { años }\end{array}$ & 201 & 53,3 \\
$\quad \leq 29$ años & 176 & 46,7 \\
Sexo & & \\
$\quad$ Hombre & 209 & 55,4 \\
$\quad$ Mujer & 168 & 44,6 \\
Región de residencia & & \\
$\quad$ Fuera de Lima y Callao & 292 & 77,5 \\
$\quad$ Lima o Callao & 85 & 22,5 \\
Estado civil & & \\
$\quad$ Casado(a) o conviviente & 205 & 54,4 \\
$\quad$ Soltero(a) o separado(a) & 172 & 45,6 \\
Instrucción alcanzada & & \\
$\quad$ Secundaria o superior completa & 321 & 85,2 \\
$\quad$ Primaria o secundaria incompleta & 56 & 14,8 \\
Uso de la información & & \\
$\quad$ Adecuado & 266 & 70,6 \\
$\quad$ Inadecuado & 111 & 29,4 \\
Afrontamiento emocional & & \\
$\quad$ Positivo & 270 & 71,6 \\
$\quad$ Negativo & 107 & 28,4 \\
\hline
\end{tabular}

Tabla 2. Prácticas de distanciamiento social en adultos que participaron de una encuesta en línea durante marzo y mayo del 2020 en Perú.

\begin{tabular}{|c|c|c|c|c|c|c|}
\hline \multirow{2}{*}{ Prácticas de distanciamiento social } & \multicolumn{2}{|c|}{ Nunca o a veces } & \multicolumn{2}{|c|}{ Frecuentemente } & \multicolumn{2}{|c|}{$\begin{array}{c}\text { Casi siempre } \\
\text { o siempre }\end{array}$} \\
\hline & $\mathrm{n}$ & $\%$ & $\mathrm{n}$ & $\%$ & $\mathrm{n}$ & $\%$ \\
\hline e mantiene alejado, extremando medidas frente a personas so & 38 & 10,1 & 116 & 30,8 & 223 & 59,2 \\
\hline vita saludar convencionalmente (abrazo, beso, dar la mano) & 48 & 12,7 & 133 & 35,3 & 196 & 52,0 \\
\hline vita salir a la calle innecesariamente, lo hace solo en casos urgentes & 48 & 12,7 & 111 & 29,4 & 218 & 57,8 \\
\hline Evita el contacto con sus vecinos, amigos, gente cercana que le rodea & 26 & 6,9 & 135 & 35,8 & 216 & 57,3 \\
\hline
\end{tabular}

Tabla 3. Factores asociados con inadecuadas prácticas de distanciamiento social en adultos que participaron de una encuesta en línea durante marzo y mayo del 2020 en Perú.

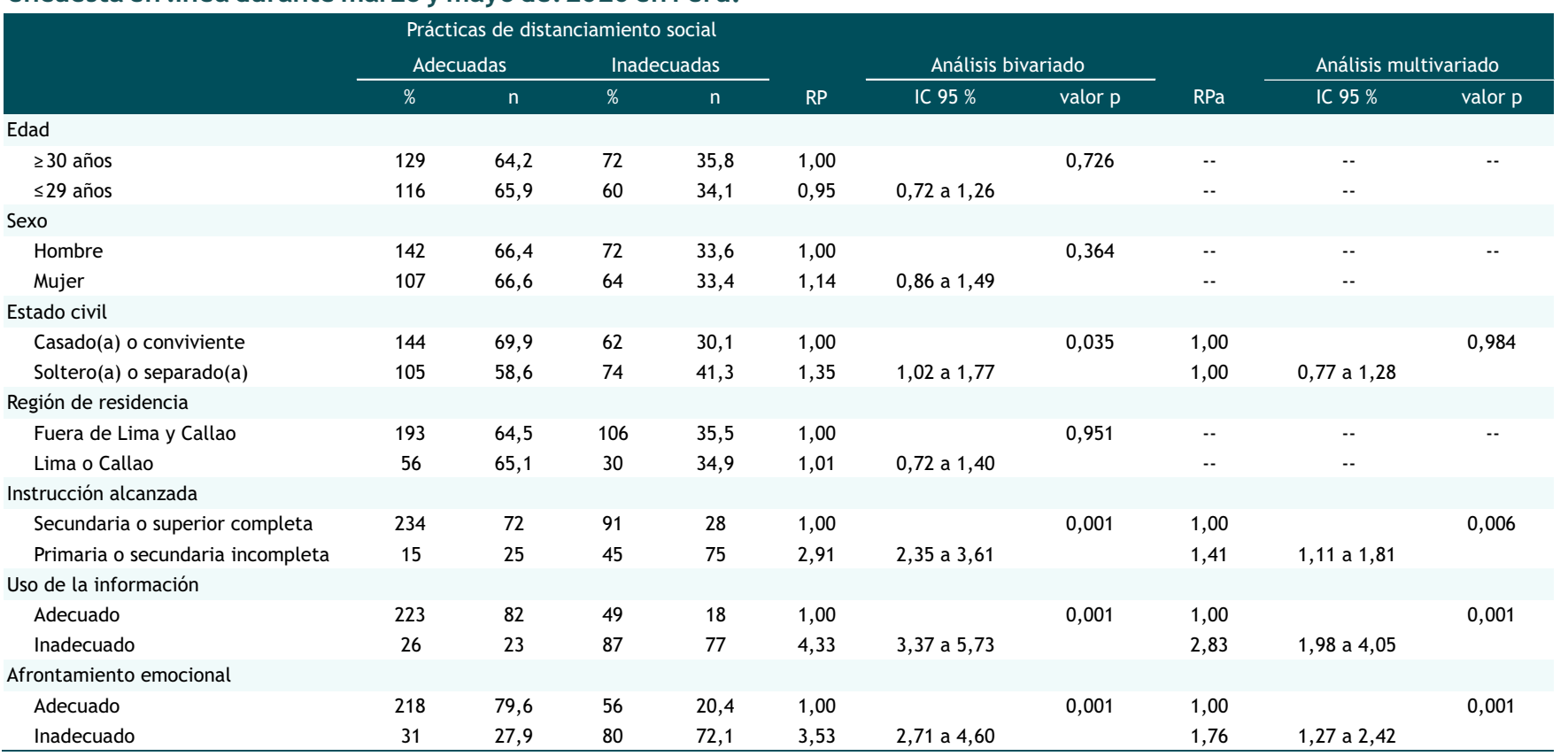


Al evaluar los factores asociados en los análisis bivariados, el estado civil soltero(a) o separado(a) (RP 1,35, IC 95\%: 1,02 a $1,77, p=0,035)$, tener grado de instrucción primaria 0 secundaria incompleta (RP 2,92, IC $95 \%: 2,35$ a 3,61), el inadecuado uso de la información (RP 4,33, IC 95\%: 3,37 a $5,73, p=0,001)$ y un negativo afrontamiento emocional (RP 3,53, IC 95\%: 2,71 a 4,69, p=0,001) fueron estadísticamente significativos e ingresaron a un análisis multivariado donde el grado de instrucción ( $R P a$ 1,41, IC 95\%: 1,11 a 1,81, $\mathrm{p}=$ 0,006 ), el inadecuado uso de la información (RPa 2,83, IC 95\% 1,98 a $4,05, p=0,001$ ) y un negativo afrontamiento emocional frente al coronavirus (RPa 1,76, IC 95\%: 1,26 a 2,42, $p=0,001$ ) se encontraron como factores asociados a inadecuadas prácticas de distanciamiento social (ver Tabla 3).

\section{DISCUSIÓN}

Los hallazgos de una encuesta en línea realizada en adultos del Perú durante un periodo de confinamiento debido a la pandemia por COVID-19 muestran que un tercio de los encuestados tuvo inadecuadas prácticas de distanciamiento social, donde tener grado de instrucción primaria o secundaria incompleta, inadecuado uso de la información o de afrontamiento emocional frente al coronavirus fueron los factores asociados.

Una encuesta aplicada a través redes sociales en Egipto encontró un $81,9 \%$ de adultos tuvo pobres prácticas de distanciamiento social, más del doble de lo reportado en el presente estudio. Los autores encontraron que algunos parámetros como el evitar las reuniones familiares y mantener suficiente espacio al tratar con otras personas fueron las principales prácticas inadecuadas ${ }^{(7)}$.

Las personas podrían obviar un distanciamiento social dentro del entorno familiar, por la falsa creencia de que los miembros de su familia están a salvo de contraer la infección, lo que puede conducir a un aumento de contagios, ya que los miembros de su familia pueden ser portadores y estar libres de síntomas. Por otro lado, en algunas regiones podría ser más difícil lograr adecuadas prácticas de distanciamiento debido a las aglomeraciones en lugares públicos, medios de transporte hacinados y la falta de estrictas regulaciones en los mercados, debido a los recursos limitados, lo que no permitiría el completo cumplimiento de las medidas de distanciamiento social. Sin embargo, ninguna intervención por si misma ofrece una protección completa y otras medidas como la higiene de las manos y el uso de mascarillas son esenciales para reducir la transmisión de la enfermedad ${ }^{(8-10)}$.

El presente estudio encontró que uno de los factores asociados con una mayor probabilidad de tener prácticas inadecuadas es el menor nivel de instrucción. Esto se podría deber a que, al ser personas sin empleos formales o con menores condiciones laborales, estarían más obligados a salir a diario para laborar en entornos con una relación más cercana con diferentes personas, como en el caso de los empleados de comercios ${ }^{(11-14)}$.

Otro factor asociado fue el inadecuado uso de la información; en ese sentido, una persona puede tomar decisiones de acuerdo con la información que recibe, por lo tanto, si no es la correcta, podría evitar cumplir o desacreditar las prácticas de distanciamiento social, que fueron sugeridas desde antes del periodo de confinamiento en Perú. En ese contexto, es conveniente proporcionar materiales educativos y de divulgación para aumentar la comprensión pública de la enfermedad e influir en el cambio de comportamiento ${ }^{(15,16)}$.

Según Fridman et al, las fuentes de información oficiales sobre la COVID-19 fueron recibidas de forma más confiable entre las personas de edad avanzada en comparación a las personas jóvenes que recurrieron mayoritariamente a las fuentes privadas y a las redes sociales; asimismo, la confianza en fuentes oficiales se asoció positivamente con la adherencia al distanciamiento social, mientras que la confianza en las redes sociales se asoció negativamente ${ }^{(15)}$.

Las fuentes oficiales o de mayor seriedad en la información que comparten sobre la COVID-19 desempeñan un rol importante en la mejora de la toma de conciencia en tiempos de una emergencia sanitaria ${ }^{(16)}$. Un estudio previo reporta que el uso de CNN (Cable News Network) como fuente de información sobre el coronavirus se asocia con una probabilidad de $10 \%$ más alta de participar en el distanciamiento social que el uso de otro canal de televisión como Fox News ${ }^{(11)}$; por lo tanto, el medio de comunicación podría tener un rol sobre la población, posteriores estudios podrían analizar más detalladamente este factor.

Asimismo, un inadecuado afrontamiento emocional frente a la pandemia, durante el periodo de confinamiento, podría incrementar la probabilidad de las prácticas inadecuadas de distanciamiento social. Esto se debería a una mayor despreocupación o no interiorización de la gravedad del problema de salud pública en su entorno, que al no generar situaciones estresantes los llevaría a mayor incumplimiento de las recomendaciones de distanciamiento social. Debido a que las medidas de confinamiento pueden ocasionar problemas a nivel de las normas sociales, la economía y el bienestar psicológico de la población ${ }^{(8,17)}$, estas han provocado precisamente cambios en el comportamiento de las personas. En el contexto de la pandemia de la COVID-19, el impacto del distanciamiento social en la salud está caracterizado por aumentos sustanciales en la ansiedad, depresión, mala calidad de sueño e inactividad física durante el periodo de aislamiento ${ }^{(17,18)}$.

Nuestro estudio tiene, varias limitaciones, una de ellas se debe al poco control sobre el método de muestreo en cadena aplicado en línea y que los encuestados compartan las mismas características; sin embargo, era la alternativa más factible debido al periodo de confinamiento establecido en todo el país, lo que impidió una mayor representatividad para la población; asimismo, el estudio no está exento de sesgos por el uso del cuestionario, la medición de las variables, y del diseño transversal por lo tanto no se podría establecer una relación de causalidad.

Se concluye que los factores que incrementaron la probabilidad de inadecuadas prácticas de distanciamiento social fueron un menor nivel de instrucción, tener inadecuado uso de la información, así como un negativo afrontamiento emocional frente a la pandemia por la COVID19 en una muestra de adultos encuestados en línea durante los meses de marzo y mayo del 2020 en Perú. 


\section{REFERENCIAS BIBLIOGRÁFICAS}

1. Qu L, Li J, Ren H. COVID-19: the epidemiology and treatment. Br J Hosp Med. 2020 [Cited 2020 dic 21]; 81(10). Doi: 10.12968/hmed.2020.0580

2. Rodríguez A, Sánchez J, Hernández S, Pérez C, Villamil W, Méndez C. Preparación y control de la enfermedad por coronavirus 2019 (COVID19) en América Latina. Acta Med Peru. 2020 [Citado 2021 Ene 10]; 37(1): 3-7. Disponible en: https: //bit.ly/3ClOgym.

3. Cetron M, Landwirth J. Public health and ethical considerations in planning for quarantine. Yale J Biol Med. 2005 [Cited 2021 feb 10]; 78(5): 329-334. Available from: https://bit.ly/3zryYGq

4. Wilder-Smith A, Freedman DO. Isolation, quarantine, social distancing and community containment: Pivotal role for old-style public health measures in the novel coronavirus (2019-nCoV) outbreak. J Travel Med. 2020; 27(2): taaa020. DOI: 10.1093/jtm/taaa020

5. Chu DL, Akl EA, Duda S, Solo K, Yaacoub S, Schünemann HJ. Physical distancing, face masks, and eye protection to prevent person-toperson transmission of SARS-CoV-2 and COVID-19: a systematic review and meta-analysis. Lancet. 2020; 395(10242): 1973-1987. DOI: $10.1016 /$ S0140-6736(20)31142-9

6. Organización Panamericana de la Salud (OPS). Enfermedades infecciosas emergentes y reemergentes. En: OPS. La salud en las Américas (Publicación Científico Técnica No. 587). Washington D.C.: OPS; 2002: 257-263.

7. Bakry HM, Waly EH. Perception and practice of social distancing among Egyptians in COVID-19 pandemic. J Infect Dev Ctries. 2020; 14(8):817-822. doi: $10.3855 /$ jidc. 13160

8. Yezli S, Khan A. COVID-19 social distancing in the Kingdom of Saudi Arabia: Bold measures in the face of political, economic, social and religious challenges. Travel Med Infect Dis. 2020; 37:101692. doi: 10.1016/j.tmaid.2020.101692

9. Lau LL, Hung N, Go DJ, Ferma J, Choi M, Dodd W, et al. Knowledge, attitudes and practices of COVID-19 among income-poor households in the Philippines: A cross-sectional study. J Glob Health. 2020; 10(1):011007. doi: 10.7189/jogh. 10.011007
10. Azlan AA, Hamzah MR, Sern TJ, Ayub SH, Mohamad E. Public knowledge, attitudes and practices towards COVID-19: A crosssectional study in Malaysia. PLoS One. 2020; 15(5):e0233668. doi: 10.1371 /journal.pone.0233668

11. Ciancio A, Kampfen F, Kohler IV, Bennett D, Bruine de Bruin W, Darling $\mathrm{J}$, et al. Know your epidemic, knowyour response: early percepcepciotn of COVID 19 and seef-reported social distancing in the United States. PLoSOne. 2020; 15(9):e0238341. DOI: 10.1371 /journal.pone. 0238341

12. Zhong BL, Luo W, Li HM, Zhang QQ, Liu XG, Li WT, et al. Knowledge, attitudes, and practices towards COVID-19 among Chinese residents during the rapid rise period of the COVID-19 outbreak: a quick online cross-sectional survey. Int J Biol Sci. 2020; 16(10):1745-1752. doi: $10.7150 /$ ijbs. 45221

13. Pedersen MJ, Favero N. Social Distancing During the COVID-19 Pandemic: Who Are the Present and Future Non-compliers? Public Adm Rev. [Internet]. 2020; 80(5):805-814. doi: 10.1111/puar.13240

14. Luna-Costa MF, Vaz de Melo J, Bof de Andrade F, Vianca SW, Macinko J. Social distancing use of fase masks and hand washing among participants in the Brazilian Lingitudinal of Aging. The Elsi-COVID-19 initiative. Cad Saude publica. 2020; 36(Sup 3):e00193920. doi: 10.1590/0102-311X00193920

15. Fridman I, Lucas N, Henke P, Zigler CK. Association between public knowledge about COVID-19 trust in information sources and adherence to social distancing cross sectional survey. JMIR Public Health Surveill. 2020; 6(3):e22060. doi: 10.2196/22060

16. Oazi A, Qazi J, Naseer K, Zeeshan M, Hardaker G, Maitama JZ, et al. Analyzing situational awareness through public opinion to predict adoption of social distancing amid pandemic COVID-19. J Med Virol. 2020; 92(7):849-855. doi: 10.1002/jmv.25840

17. Galea S, Merchant RM, Lurie N. The Mental Health Consequences of COVID-19 and Physical Distancing: The Need for Prevention and Early Intervention. JAMA Intern Med. 2020; 180(6):817-818. doi: 10.1001/jamainternmed.2020.1562

18. Sepúlveda-Loyola W, Rodríguez-Sánchez I, Pérez-Rodríguez P, Ganz F, Torralba R, Oliveira DV, et al. Impact of Social Isolation Due to COVID19 on Health in Older People: Mental and Physical Effects and Recommendations. J Nutr Health Aging. [Internet]. 2020; 24(9):938947. doi: $10.1007 / \mathrm{s} 12603-020-1469-2$. 